\title{
Using rapid point-of-care tests to inform antibiotic choice to mitigate drug resistance in gonorrhoea
}

Carolin Vegvari ${ }^{1}$, Yonatan H Grad ${ }^{2,3}$, Peter J White ${ }^{1,4,5}$, Xavier Didelot ${ }^{1,4,6}$, Lilith K Whittles ${ }^{1,4}$, Nicole E Scangarella-Oman ${ }^{7}$, Fanny S Mitrani-Gold ${ }^{7 *}$, Etienne Dumont ${ }^{7}$, Caroline R Perry ${ }^{7}$, Kim Gilchrist ${ }^{7,8}$, Mohammad Hossain ${ }^{7}$, Tatum D Mortimer ${ }^{2}$, Roy M Anderson ${ }^{1}$, David Gardiner ${ }^{7}$

1. Department of Infectious Disease Epidemiology, School of Public Health, Imperial College London, London, United Kingdom

2. Department of Immunology and Infectious Diseases, Harvard T.H. Chan School of Public Health, Boston, United States

3. Division of Infectious Diseases, Brigham and Women's Hospital, Harvard Medical School, Boston, United States

4. MRC Centre for Global Infectious Disease Analysis and NIHR Health Protection Research Unit in Modelling and Health Economics, School of Public Health, Imperial College London, London, United Kingdom

5. Modelling and Economics Unit, National Infection Service, Public Health England, London, United Kingdom

6. Current affiliation: School of Life Sciences and Department of Statistics, University of Warwick, United Kingdom

7. GlaxoSmithKline, Collegeville, Pennsylvania, United States

8. Current affiliation: Pfizer, Inc, Pennsylvania, United States

Correspondence: Carolin Vegvari (c.vegvari@imperial.ac.uk)

Citation style for this article:

Vegvari Carolin, Grad Yonatan H, White Peter J, Didelot Xavier, Whittles Lilith K, Scangarella-Oman Nicole E, Mitrani-Gold Fanny S, Dumont Etienne, Perry Caroline R, Gilchrist Kim, Hossain Mohammad, Mortimer Tatum D, Anderson Roy M, Gardiner David . Using rapid point-of-care tests to inform antibiotic choice to mitigate drug resistance in gonorrhoea. Euro Surveill. 2020;25(43):pii=1900210. https://doi.org/10.2807/1560-7917.ES.2020.25.43.1900210

Background: The first cases of extensively drug resistant gonorrhoea were recorded in the United Kingdom in 2018. There is a public health need for strategies on how to deploy existing and novel antibiotics to minimise the risk of resistance development. As rapid point-of-care tests (POCTs) to predict susceptibility are coming to clinical use, coupling the introduction of an antibiotic with diagnostics that can slow resistance emergence may offer a novel paradigm for maximising antibiotic benefits. Gepotidacin is a novel antibiotic with known resistance and resistancepredisposing mutations. In particular, a mutation that confers resistance to ciprofloxacin acts as the 'stepping-stone' mutation to gepotidacin resistance. Aim: To investigate how POCTs detecting Neisseria gonorrhoeae resistance mutations for ciprofloxacin and gepotidacin can be used to minimise the risk of resistance development to gepotidacin. Methods: We use individual-based stochastic simulations to formally investigate the aim. Results: The level of testing needed to reduce the risk of resistance development depends on the mutation rate under treatment and the prevalence of stepping-stone mutations. A РОСТ is most effective if the mutation rate under antibiotic treatment is no more than two orders of magnitude above the mutation rate without treatment and the prevalence of stepping-stone mutations is $1-13 \%$. Conclusion: Mutation frequencies and rates should be considered when estimating the POCT usage required to reduce the risk of resistance development in a given population. Molecular POCTs for resistance mutations and stepping-stone mutations to resistance are likely to become important tools in antibiotic stewardship.

\section{Introduction}

Neisseria gonorrhoeae, the causal agent of the sexually transmitted infection (STI) gonorrhoea, is becoming increasingly resistant to available antibiotic treatment options $[1,2]$. The most widely recommended treatment for gonorrhoea is a combination therapy of ceftriaxone plus azithromycin, administered empirically without bacterial culture or point-of-care testing [3]. In isolates collected across Europe, the proportion of isolates with decreased susceptibility to ceftriaxone increased from $15 \%$ to $17.7 \%$ from 2015 to 2016 . At the same time azithromycin resistance across Europe was stable at about $7 \%$ but was much higher in individual countries (34.5\% in Portugal) [4]. The first treatment failure of this dual therapy was reported in the United Kingdom (UK) in 2014 [5]. Azithromycin resistance in combination with reduced susceptibility to ceftriaxone has been well-studied [4]. Resistance to previous recommended treatments, such as ciprofloxacin, is generally high (30-70\% in Europe, above $70 \%$ in East Asia) [2]. As ceftriaxone is at the same time the first-line and lastresort treatment, the World Health Organization (WHO) in 2017 declared the possible evolution of untreatable gonorrhoea a global public health emergency [6].

In an attempt to spare ceftriaxone as a last-resort treatment, rapid point-of-care tests (POCTs) detecting ciprofloxacin resistance mutations have been developed. Thus, even though ciprofloxacin is no longer recommended for gonorrhoea treatment, it could still be used when a POCT detects no resistance mutations [7]. Such tests could easily be expanded to include known resistance markers for other antibiotics. 
Genotypes of isolates at baseline and test-of-cure from gepotidacin treatment failures with emergence of resistance, phase II clinical trial, 2017 [11]

\begin{tabular}{|l|c|c|c|c|c|}
\hline \multirow{2}{*}{ Participant number } & Visit & Genotype gyrA & Genotype parC & MIC gepotidacin (mg/L) & $\begin{array}{c}\text { MIC ciprofloxacin } \\
\text { (mg/L) }\end{array}$ \\
\hline \multirow{2}{*}{4} & Baseline & S91F D95G & D86N & 1 & 8 \\
\cline { 2 - 6 } & Test-of-cure & S91F A92T D95G & D86N & $>32$ & 8 \\
\hline \multirow{2}{*}{6} & Baseline & S91F D95G & D86N & 1 & 4 \\
\cline { 2 - 6 } & Test-of-cure & S91F A92T D95G & D86N & 32 & 4 \\
\hline
\end{tabular}

MIC: minimum inhibitory concentration.

Mutation gyrA A92T leading to gepotidacin resistance is displayed in bold.

Gepotidacin is a novel topoisomerase IIA inhibitor currently under development and in phase III clinical trials with activity against $N$. gonorrhoeae [8]. Its mechanism of action differs from that of fluoroquinolones, and it has demonstrated activity against most ciprofloxacinresistant gonococcal strains [9]. Ciprofloxacin inhibits bacterial DNA gyrase and topoisomerase IV. The main ciprofloxacin resistance mutations in genes coding for DNA gyrase subunit A (GyrA) and topoisomerase IV subunit $\mathrm{A}$ (ParC) in $N$. gonorrhoeae are presented in the Supplementary Material 1, Supplementary Table 1. In a recent phase II clinical trial on the efficacy of gepotidacin against uncomplicated genitourinary gonorrhoea, emergence of resistance was observed for $N$. gonorrhoeae isolates from two treatment failures following use of a single dose of $3 \mathrm{~g}$ gepotidacin. This resistance is likely to have emerged due to the combination of a pre-existing ciprofloxacin resistance mutation (D86N) in the parC gene and de novo within-host emergence of an A92T mutation in the gyrA gene (Table 1). Additional experiments suggest that the gepotidacin minimum inhibitory concentration (MIC) is only significantly increased if both mutations are present together [10]. See Supplementary Material 1, Supplementary Text 1 for more details on the microbiological analysis of the phase II clinical trial. Structural analysis of the interaction of gepotidacin with GyrA suggests that gepotidacin does not interact with the two quinolone binding sites in GyrA at amino acid positions 91 and 95 [9]. Therefore, it was assumed that the S91F and D95G mutations in gyrA were not critical for the evolution of gepotidacin resistance. There may be other mutations that can cause resistance to gepotidacin, but they were not observed in the phase II clinical trial.

Novel post-treatment mutations occurred in isolates from two subjects that were treated with a single dose of 39 gepotidacin [11]. The mutations $\mathrm{S} 91 \mathrm{~F}$ and $\mathrm{D} 95 \mathrm{G}$ in gyrA and D86N in parC on their own confer different levels of resistance to ciprofloxacin.

Here, we consider the novel paradigm of introducing an antibiotic together with a POCT to control gonococcal infections and slow down resistance development. A POCT for gepotidacin resistance would determine if the known stepping-stone mutations, gyrA A92T or parC D86N, were present. If neither were detected, then gepotidacin could be used without substantial risk of treatment failure, based on current evidence, as there are no other known clinically relevant targetspecific resistance mutations for gepotidacin in $N$. gonorrhoeae. If one or both mutations were present, treatment with another antibiotic would be indicated.

Determining the frequencies of resistance mutations requires surveillance systems such as the data recorded in the European Gonococcal Antimicrobial Surveillance Programme (Euro-GASP, data collection since 2009) and the US Gonococcal Isolate Surveillance Project database (GISP, data collection since 1986). Euro-GASP monitors $N$. gonorrhoeae antimicrobial susceptibility trends by phenotypically characterising isolates from male and female patients. GISP samples isolates from male patients attending STD clinics. Each participating country contributes 100 cultured and characterised isolates per year.

Our study aims to answer several questions using a theoretical modelling framework: can a molecular POCT that detects known stepping-stone mutations prevent the spread of gepotidacin-resistant strains? Under what conditions is a POCT most effective at reducing the risk of resistance development, and how frequently would such a test need to be used to reduce this risk by at least $50 \%$ over five years? These questions have broader implications for designing antibiotic stewardship strategies and prolonging the life span of novel and existing antibiotics.

\section{Methods}

\section{Model framework}

We developed a compartmental deterministic model framework of gonorrhoea transmission building on previous models $[12,13]$. As in Whittles et al. [14] our model uses transmission parameter values derived from men who have sex with men (MSM) populations in London. The model has three compartments, susceptible (S), infected (I) and treated (T) individuals (Figure 1, Supplementary Material 1, Supplementary Text 2). Individuals in the infected class are infected but not currently treated. Individuals in the treated class are infected and currently receiving treatment. The time 
FIGURE 1

Two-locus gonorrhoea antibiotic resistance model

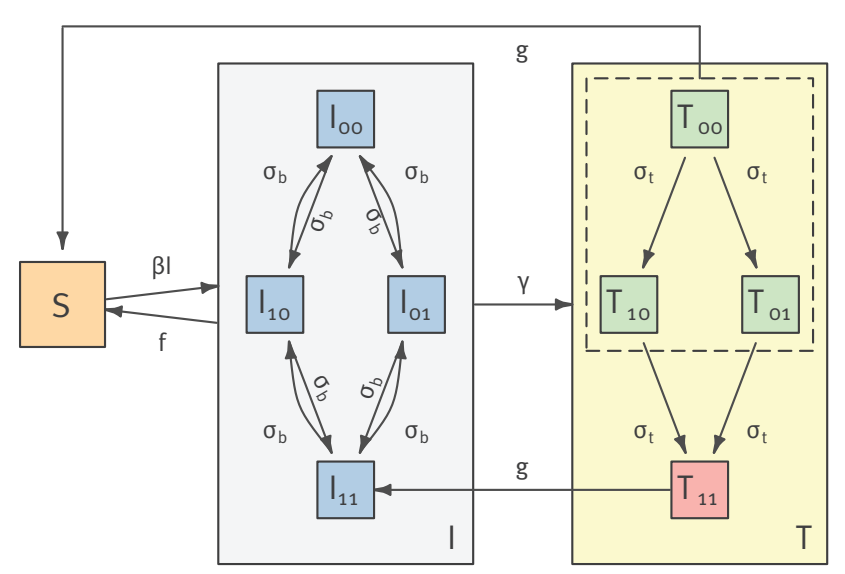

S: susceptible individuals; I: infected, untreated individuals; T: infected, treated individuals.

The basic model has three main compartments, susceptible, infected and treated individuals (i.e. infected individuals that are undergoing treatment). Individuals can be infected with one of four gonococcal strains that differ in the combination of resistance ('stepping-stone') mutations they carry (0o, 10, 01 or 11). Only strains with both gepotidacin resistance mutations (11) are considered resistant to gepotidacin. Strains with one resistance mutation (10 and 01) are resistant to ciprofloxacin, but sensitive to gepotidacin. First subscript: state of resistance for the first locus (o - sensitive, 1 - resistant); second subscript: state of resistance for the second locus (o - sensitive, 1 - resistant); $\beta$ : infection rate parameter (product of sexual contact rate and infection probability per contact); f: spontaneous recovery rate from infection without treatment ( $1 / f-$ duration of natural infection); $g$ : Cure rate in individuals currently being treated ( $1 / \mathrm{g}$ - duration of treatment); $\gamma$ : treatment seeking rate $(1 / \gamma-$ time from start of infection to start of treatment); $\sigma_{b}$ : locus-specific mutation rate in untreated individuals (no selection pressure); $\sigma_{t}$ : locus-specific mutation rate in treated individuals (under selection pressure).

that individuals spend in the treated class is the duration for which the within-host antibiotic concentration is great enough to clear the infection.

The key novel feature of our model is that we explicitly consider different resistance genotypes of relevance to gepotidacin. Given that there are two known steppingstone mutations that together cause elevated MICs, susceptible individuals can be infected by one of four strains. If o signifies the sensitive allele and 1 the resistant allele, these strains are: 00, 10, 01 and 11. Based on the observations from the phase II clinical study [15], we assume that only the 11 genotype is resistant to both ciprofloxacin and gepotidacin, whereas 10 and 01 are resistant to ciprofloxacin only. The model also allows for the possibility that both resistance mutations can arise de novo over the course of an infection.

\section{Model parameters and transitions}

Susceptible individuals become infected at rate $\beta$, which in our modelling framework is population-specific and depends on the sexual contact rate and the infection probability per contact (Table 2). Infected individuals seek treatment at rate $\gamma$ and can recover spontaneously at rate f. Treated individuals recover at rate $g$ if they are not resistant, and return to the susceptible class. Individuals with treatment failures are reclassified as infected. For more details on the model processes see Supplementary Material 1, Supplementary Text 2. To assess the impact of uncertainty in model parameters, we performed sensitivity analyses by varying model parameters across a range of measured values from the literature. We assume an annual incidence rate of gonorrhoea of 22,000 cases in a total population of 1.5 million individuals, approximating the MSM population in the UK [16]. We varied the starting conditions for each of the simulation scenarios described below. For model parameter and variable values used in the simulations see Table 2 .

\section{Resistance evolution}

Sensitive strains can acquire resistance to antibiotics by de novo mutations. The mutation rate in $N$. gonorrhoeae has been determined from phylogenetic studies [17]. Several studies indicate that the mutation rate under treatment may be increased due to the SOS DNA damage response [18-20]. A DNA damage response system in N. gonorrhoeae has been described by Schook et al. [21]. Other topoisomerase II inhibitors are known to increase the mutation rate by interfering with DNA replication [22,23]. However, as the mechanism of action of gepotidacin differs from that of conventional topoisomerase II inhibitors, it may not increase the mutation rate to the same extent. There are no estimates for mutation rates in $N$. gonorrhoeae under antibiotic pressure. We therefore performed simulations for a range of mutation rate parameters under treatment based on estimates obtained from other bacterial species (Table 2).

N. gonorrhoeae is known to have a high rate of homologous recombination [24]. Recombination between different gonococcal strains can only occur in mixed infections at the same anatomical site. Thus, the effective recombination rate can be calculated as:

coinfection frequency $\times$ ratio of recombination to mutation $\times$ base mutation rate

The coinfection frequency with different gonococcal strains at the same anatomical site is unknown, but we can use the frequency of infections with different gonococcal strains at different anatomical sites as a proxy upper-bound estimate for the frequency of mixed infections (13\%) at the same anatomical site [25]. The ratio of recombination to mutation events has been estimated from whole genome sequence data (genomewide average) $[17,26,27]$. If we assume a mutation rate of $2.45 \times 10^{-8}$ substitutions per nt per day and a recombination-to-mutation ratio of 2.2 [27], we obtain an effective recombination rate of $7 \times 10^{-9}$. Since this would lead to an increase in the rate of resistance acquisition that is smaller than the increased mutation rates that we tested, we do not explicitly consider recombination in the model. 
TABLE 2

Parameter values used in simulation model

\begin{tabular}{|c|c|}
\hline Model parameter (unit) & Values used in individual simulations \\
\hline Infection rate (per day) & $\begin{array}{c}5.56 \times 10^{-8}, 1.67 \times 10^{-8}, 6.02 \times 10^{-8}, 2.28 \times 10^{-7} \\
2.29 \times 10^{-7}\end{array}$ \\
\hline Recovery rate $\mathrm{f}$ (inverse of duration of natural infection) (per day) & $1 / 84,1 / 160,1 / 185,1 / 240,1 / 365$ \\
\hline $\begin{array}{l}\text { Treatment rate } y \text { (inverse of time in days until patients first seek treatment) (per } \\
\text { day) }\end{array}$ & $1 / 3,1 / 12,1 / 13,1 / 52$ \\
\hline $\begin{array}{l}\text { Cure rate for gepotidacin treatment, assuming double dose (inverse of } \\
\text { treatment duration, i.e. time over MIC) (per day) }\end{array}$ & $1.778(=1 / 13.5 \mathrm{~h})$ \\
\hline $\begin{array}{l}\text { Cure rate for ciprofloxacin treatment, assuming single dose (inverse of } \\
\text { treatment duration) (per day) }\end{array}$ & $6(=1 / 4 \mathrm{~h})$ \\
\hline Proportion of patients that return for second round treatment $p$ & $1,0.8,0.6,0.5$ \\
\hline Mutation rate without treatment $\sigma_{b}$ (substitutions per nt per day) & $3.12 \times 10^{-9}, 2.45 \times 10^{-8}$ \\
\hline Mutation rate with treatment $\sigma_{t}$ (substitutions per nt per day) & $\begin{array}{c}3.12 \times 10^{-9}, 2.45 \times 10^{-8}, 4.9 \times 10^{-8}, 1.23 \times 10^{-7}, 2.45 \times 10^{-7}, \\
2.45 \times 10^{-6}, 2.45 \times 10^{-5}, 7.95 \times 10^{-5}, 9.66 \times 10^{-4}\end{array}$ \\
\hline Point-of-care test usage (\%) & $0,10,20,30,40,50,60,70,80,90,100$ \\
\hline Total simulated population & $1.5 \times 10^{6}$ \\
\hline Initial number of infected individuals/equilibrium incidence rate & 22,000 \\
\hline Initial prevalence of parC D86N (\%) & $\begin{array}{c}0,0.06,0.18,0.462,0.669,1.5,2,2.9,3,5.9,6.5,8.6,13, \\
19.3,38.6\end{array}$ \\
\hline Initial prevalence of $g y r A$ A92T (\%) & 0,1 \\
\hline Initial prevalence of double mutant (parC D86N/gyrA A92T) (\%) & o \\
\hline
\end{tabular}

MIC: minimum inhibitory concentration.

All rates are per day. If more than one value is given, the whole range of values has been tested in different simulations. See Supplementary Material 2 for parameter combinations used in individual simulations. References and the basis of assumptions are included in the Supplementary Material 1, Supplementary Table 2.

\section{Treatment scenarios and outcome measure} In our numerical evaluations of the model of a POCT detecting resistance mutations we varied the use of the POCT as a proportion of treated gonorrhoea infections from $0 \%$ to $100 \%$ and the assumed sensitivity and specificity of the test from 80 to $100 \%$. If a POCT was used then gepotidacin was only used as a treatment if no resistance mutations were detected. If no POCT was used then gepotidacin was used as a firstline treatment.

We used stochastic simulations based on the deterministic structure defined in Supplementary Material 1, Supplementary Text 2, Figure S1, Equations 2, Table S1, and a Gillespie algorithm to analyse model behaviour and predictions. We recorded the number of simulations out of 100 replications in which the $5 \%$ resistance threshold was reached at any time point over a five-year timeframe. (This corresponds to the WHO recommendation that when resistance to a specific antibiotic exceeds $5 \%$, alternative antibiotics should be used [28].) Table 2 lists parameter values used in simulations. A full list of parameter combinations used in each simulation scenario together with the results can be found in Supplementary Material 2.

Determining the prevalence of parC $\mathrm{D} 86 \mathrm{~N}$ in Europe and the United States

We obtained publicly available $N$. gonorrhoeae whole genome sequencing (WGS) data from the National
Center for Biotechnology Information (NCBI) Sequence Read Archive deposited as part of the studies in Supplementary Material 1, Supplementary Table 3. We ran FastQC [29] to assess WGS data quality and removed accessions with insufficient or poor-quality reads. We mapped reads to $N$. gonorrhoeae NCCP11945 (NC_011035.1) using BWA-MEM vs 0.7.17-r1188 [30]. Duplicates were marked using Picard vs 2.8.0 (https:// github.com/broadinstitute/picard). We called variants using Pilon vs 1.23 [31] with minimum depth of $10 \mathrm{X}$ and minimum mapping quality of 20 . We removed accessions where more than $15 \%$ of sites were unable to be called by Pilon due to insufficient coverage or poor mapping quality. We identified variants in gyrA and parC corresponding to the amino acid mutations gyrA A92T and parC D86N.

Currently, no published genomic databases report frequencies for the $g y r A$ A92T mutation. The highest reported prevalence of the parC D86N mutation was $38.6 \%$ of ciprofloxacin-resistant isolates [12]. We genotyped 10,259 unique accessions that passed our quality control filters. The frequencies of parC D86N and gyrA A92T mutations are low in Europe and in the United States (Euro-GASP: parC D86N $1.8 \%$, gyrA A92T ०\% of all gonococcal isolates analysed September-November 2013 [32], GISP: parC D86N $0.635 \%$, gyrA A92T 0\%, of all gonococcal isolates analysed 2000-2013 [33]). This means that in Europe 
Proportion of simulations in which the frequency of gepotidacin-resistant strains reaches $5 \%$ with different mutation rates, prevalence of parC D86N and POCT usage levels
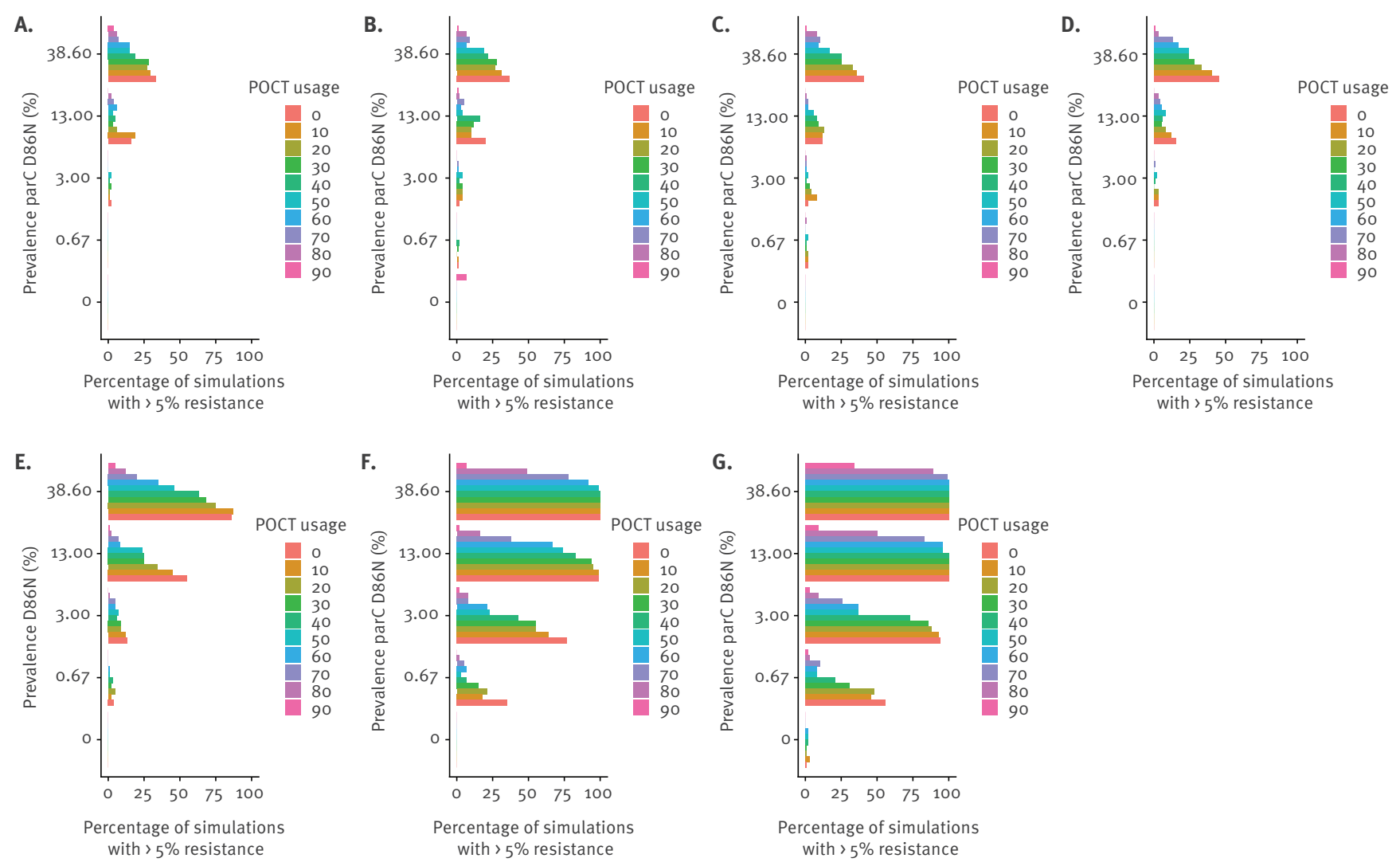

POCT: point-of-care testing.

The initial prevalence of gyrA A92T was o in all simulations. Mutation rates (substitutions per nt per day): A. $2.45 \times 10^{-8}, \mathrm{~B} .4 .9 \times 10^{-8}, \mathrm{C}$. $1.23 \times 10^{-7}$, D. $2.45 \times 10^{-7}$, E. $2.45 \times 10^{-6}$, F. $2.45 \times 10^{-5}$, G. $7.95 \times 10^{-5}$. Total number of simulations 100 .

and the US simulation assuming $0.6-6.5 \%$ initial prevalence of parC D86N are the most applicable.

\section{Ethical statement}

No ethical approval was required for this study because no new data have been collected as part of the study.

\section{Results}

If the mutation rate with treatment is the same as without treatment, then even a POCT usage of $20-30 \%$ can reduce the risk of resistance development (Figure $2 \mathrm{~A})$. With assumptions of complete testing and perfect sensitivity and specificity, resistance did not develop in our simulations. If the initial prevalence of the stepping-stone mutations was lower than $6 \%$, stochastic effects were important, so that even high POCT usage had little impact on the emergence of resistant strains (Supplementary Material 1, Supplementary Figure 1). In populations with an initial frequency of more than $6 \%$ of the parC D86N mutation, a POCT had a potential to reduce the risk of resistance reaching $5 \%$. The effect of the POCT was roughly proportional to the usage level.
The greater the mutation rate during treatment and the higher the population prevalence of stepping-stone mutations, the higher the POCT usage needed to reduce the risk of resistance development (Figure 2). With an initial frequency of the parC D86N greater than $10 \%$ and a mutation rate during treatment of more than 1,000 times the baseline mutation rate, POCT usage had to be $80-90 \%$ to halve the risk of resistance development (Figure $2 \mathrm{G}$ ). An increase in mutation rate during treatment of this order of magnitude is rarely observed in laboratory experiments (Supplementary Material 1, Supplementary Table 4). If the initial prevalence of gyrA A92T is $1 \%$, rather than $0 \%$, more resistance emerges, especially if mutation rates under treatment are high (Supplementary Material 1, Supplementary Figure 2). If the mutation rate under treatment is less than 1,000 times the baseline mutation rate, the added risk of resistance development from gyrA A92T prevalence of $1 \%$ stays below $10 \%$.

If there were no stepping-stone mutations in a population, the risk of resistance development was generally 
low ( $<5 \%$ if the prevalence of parC D86N was $<13 \%$ and only exceeding $25 \%$ if the prevalence of parC D86N was $38.6 \%$ ), and a POCT was only required if the mutation rate during treatment was very high $\left(9.66 \times 10^{-4}\right.$ per site per day) (Supplementary Material 1, Supplementary Figures 1,3$)$. The sensitivity and specificity of the test did not have a great influence on the risk of resistance development in the range tested. With high POCT usage $(70-90 \%)$, a higher sensitivity (99\% compared to $80 \%$ ) of the test slightly decreases the probability of resistance spreading (Supplementary Material 1, Supplementary Figure 4).

\section{parC D86N prevalence}

We did not observe any isolates with gyrA A92T. We found that across all datasets $6.1 \%(626 / 10,259)$ of isolates encoded the parC D86N mutation. parC D86N was observed in 17 of 20 datasets.

If the mutation rate is not increased under treatment, the risk of resistance emergence is less than $5 \%$ in scenarios assuming $0.6-6.5 \%$ initial prevalence of parC D86N.

\section{Discussion}

Our results indicate that a molecular POCT detecting the two known stepping-stone mutations implicated in gepotidacin resistance could help reduce the risk of resistance development to gepotidacin, a novel antibiotic undergoing phase III trials, by N. gonorrhoeae. The ability to do so would depend on the population prevalence of stepping-stone mutations and the mutation rate under treatment. If both are low, then most strains will be sensitive to gepotidacin and a POCT would have a negligible effect on the risk of resistance development. If the mutation rate under treatment is very high and a large proportion of strains already have one stepping-stone mutation, a POCT would not be able to prevent resistance spreading, because resistance would arise too frequently after testing in previously sensitive infections. A high rate of horizontal gene transfer between coinfecting strains could equally lead to increased rates of resistance emergence [34]. It is possible that other fluoroquinolone resistance mutations affect the MIC for gepotidacin in N. gonorrhoeae, but none have so far been identified.

This suggests that a POCT would be most valuable if the increase in mutation rate under treatment is moderate (no more than 100 times above the baseline mutation rate) and the prevalence of pre-existing resistance mutations is at least $1 \%$. In this case and if the prevalence of resistance mutations is not too high (maximum $13 \%$ in our analyses), even a $20-30 \%$ usage of the POCT could, given our assumptions, halve the risk of resistance development. This would be the case for all publicly available datasets we surveyed, where $6.1 \%$ of all $N$. gonorrhoeae genomes carry parC D86N. However, due to stochastic variability, $50 \%$ usage would be preferable to reliably halve the risk of resistance development. The prevalence of parC D86N is expected to vary among different countries. Therefore, optimum POCT usage values will be country-specific.

These results are in good agreement with a recent study which found that a POCT that detects resistance to three antibiotics used to treat gonorrhoea can prevent resistant strains from spreading, if its usage is at least $37 \%$, and that test sensitivity and specificity have a minor effect on resistance development [35]. Our study also agrees with results from Fingerhuth et al. according to which a POCT test with resistance detection prevents more cases of antibiotic-resistant gonorrhoea than a NAAT test without resistance detection, unless the POCT sensitivity is lower than $80 \%$ [36].

Since gepotidacin resistance only arises when both known stepping-stone mutations occur in the same strain, the relationship between the mutation rate under treatment and the risk of resistance development is not linear. Small increases in mutation rate of up to 10 -fold did not increase the risk of resistance development in our simulations, unless the initial prevalence of parC D86N was assumed to be greater than $30 \%$. If the mutation rate under treatment increased 1,000 times or more, resistance almost always developed within 5 years.

Mutation rates during antibiotic exposure of this magnitude are rare according to the literature on other bacterial species (Supplementary Material 1, Supplementary Table 4). Moreover, mutation rate measurements from in vitro experiments are prone to overestimation [37]. Our results suggest that estimates of the mutation rate under antibiotic exposure should be taken into account when evaluating treatment strategies. For example, Obolski and Hadany use a simulation model to show that in hospitals antibiotic mixing and cycling are superior to combination therapy, if bacterial mutagenesis is stress-induced [38].

We did not consider fitness costs of antibiotic resistance mutations, because there is no population-level data on potential fitness costs of gepotidacin resistance mutations. As fluoroquinolone-resistant strains persist in the population, we can assume that fitness costs associated with fluoroquinolone-resistance mutations are small or absent [33]. Our model represents a worst-case scenario regarding the speed of spread of gepotidacin resistance. If there were sufficiently high fitness costs associated with one or both known stepping-stone mutations leading to gepotidacin resistance and a POCT could ensure that only infections without stepping-stone mutations were treated with gepotidacin, then newly-arising gepotidacin-resistant strains would potentially quickly become extinct [14].

Since some of the data for this study came from a relatively small sample (a phase II clinical trial), the evaluation may have to be updated when more data becomes available. In the case of treatment failure, the sequence in which alternative antibiotics are 
prescribed can matter, especially if there is evidence for cross-resistance or resistance mutations to different antibiotics for the same strains. Therapies with multiple targets, or antibiotics that require multiple mutations before they lose their efficacy, should be preferred as first-line treatments.

The main limitation of this study is the lack of empirical information on key model parameters. For example, estimates for the duration of natural infection are based on limited observational studies from before 1980. Similarly, the duration from infection to when patients seek treatment may vary among different populations. The population we model approximates an MSM population and likely overestimates treatment rates for women who are more frequently asymptomatic. However, as long as we compare simulation scenarios with the same sets of parameters, the qualitative outcome of our analysis is unlikely to change. Another limitation is that potentially we do not know all mechanisms of resistance to gepotidacin and we acknowledge the need for genomic surveillance to determine if other resistance mutations can arise.

Simulation studies can inform us on what data should be collected to improve treatment strategies. In the case of gepotidacin, molecular surveillance data to estimate the frequency of known stepping-stone mutations is required. More generally, whole-genome surveillance data in combination with phenotypic antimicrobial susceptibility data can inform us about the frequency of resistance genes to other antimicrobials used for gonorrhoea treatment. In vitro or animal model experiments could help to estimate the mutation rate under gepotidacin exposure. Mutation prevalence and rate should be considered when estimating the POCT usage required to reduce the risk of resistance development in a given population. Molecular POCTs for resistance mutations and stepping-stone mutations are likely to become important tools in antibiotic stewardship and surveillance in the coming years, and a combination of empirical study and modelling is required to optimise their use for public health benefit.

\section{* Erratum}

The affiliation of Fanny S Mitrani-Gold was corrected on 6 Nov 2020, after publication of the article.

\section{Disclaimer}

The views expressed are those of the authors and not necessarily those of the Department of Health and Social Care; the Foreign, Commonwealth \& Development Office; MRC; NIHR; NHS; or Public Health England.

\section{Acknowledgements}

The authors would like to thank Brian Spratt for helpful discussions on the work presented in this manuscript.

\section{Funding}

This study was supported by GlaxoSmithKline and was funded in part with Federal funds from the Office of the Assistant Secretary for Preparedness and Response, Biomedical Advanced Research and Development Authority (BARDA), under agreement \# HHSO100201300011C.

CV was funded by a research grant (3000032222) and YHG by consulting fees from GlaxoSmithKline (GSK). PJW and XD were funded by the UK National Institute for Health Research (NIHR) Health Protection Research Unit (HPRU) in Modelling Methodology at Imperial College London in partnership with Public Health England (PHE) (grant HPRU-2012-10080). PJW and LKW acknowledge funding from the UK Medical Research Council (MRC) Centre for Global Infectious Disease Analysis (MR/R015600/1); this award is jointly funded by the MRC and the UK Foreign, Commonwealth \& Development Office (FCDO) under the MRC/FCDO Concordat agreement and is also part of the EDCTP2 programme supported by the European Union (EU). TDM was funded by the Smith Family Foundation and NIH NIAID grant (F32Al145157). GSK authors contributed to study design, provided data and were involved in the interpretation of data and results. The Department of Health and Social Care; Foreign, Commonwealth \& Development Office; MRC; NIHR; Public Health England; NIH and the Smith Family Foundation had no role in study design, data collection and analysis, decision to publish, or preparation of the manuscript.

\section{Conflict of interest}

PJW, XD, LKW and TDM have no competing interests and have received no funding from GSK for this work. CV received funding from GSK for this work. NESO, FSMG, ED, CRP, MH, DG are employees of GSK. RMA was a non-executive board member of GSK till June 2018. YHG has consulted for GSK. KG is an employee for Pfizer and was formerly an employee of GSK.

\section{Authors' contributions}

Study conceptualisation and design: CV, YHG, PJW, XD, LKW, NESO, FSMG, ED, CRP, KG, MH, RMA, DG.

Conducting the analyses: CV, YHG, TDM.

Writing the original manuscript: CV.

Providing data for the analysis: NESO, FSGM, ED, CRP, KG, MH, DG, YHG, TDM.

Editing and approving the manuscript: CV, YHG, PJW, XD, LKW, NESO, FSMG, ED, CRP, KG, MH, TDM, RMA, DG.

\section{References}

1. Unemo M. Current and future antimicrobial treatment of gonorrhoea - the rapidly evolving Neisseria gonorrhoeae continues to challenge. BMC Infect Dis. 2015;15(1):364. https://doi.org/10.1186/s12879-015-1029-2 PMID: 26293005

2. Wi T, Lahra MM, Ndowa F, Bala M, Dillon JR, Ramon-Pardo P, et al. Antimicrobial resistance in Neisseria gonorrhoeae: Global surveillance and a call for international collaborative action. PLoS Med. 2017;14(7):e1002344. https://doi.org/10.1371/ journal.pmed.1002344 PMID: 28686231

3. World Health Organization (WHO). WHO Guidelines for the Treatment of Neisseria gonorrhoeae. Geneva: WHO. 2016. Available from: https://www.who.int/reproductivehealth/ publications/rtis/gonorrhoea-treatment-guidelines/en/ 
4. Day MJ, Spiteri G, Jacobsson S, Woodford N, Amato-Gauci AJ, Cole MJ, et al. Stably high azithromycin resistance and decreasing ceftriaxone susceptibility in Neisseria gonorrhoeae in 25 European countries, 2016. BMC Infect Dis. 2018;18(1):609. https://doi.org/10.1186/s12879-018-3528-4 PMID: 30509194

5. Fifer H, Natarajan U, Jones L, Alexander S, Hughes G, Golparian $D$, et al. Failure of Dual Antimicrobial Therapy in Treatment of Gonorrhea. N Engl J Med. 2016;374(25):2504-6. https://doi. org/10.1056/NEJMC1512757 PMID: 27332921

6. World Health Organization (WHO). Global priority list of antibiotic-resistant bacteria to guide research, discovery, and development of new antibiotics. Geneva: WHO. 27 Feb 2017. Available from: https://www.who.int/medicines/publications/ global-priority-list-antibiotic-resistant-bacteria/en/

7. Allan-Blitz LT, Humphries RM, Hemarajata P, Bhatti A, Pandori MW, Siedner MJ, et al. Implementation of a Rapid Genotypic Assay to Promote Targeted Ciprofloxacin Therapy of Neisseria gonorrhoeae in a Large Health System. Clin Infect Dis. 2017;64(9):1268-70. PMID: 28034887

8. Farrell DJ, Sader HS, Rhomberg PR, Scangarella-Oman NE, Flamm RK. In Vitro Activity of Gepotidacin (GSK2140944) against Neisseria gonorrhoeae. Antimicrob Agents Chemother. 2017;61(3):e02047-16. https://doi.org/10.1128/AAC.02047-16 PMID: 28069643

9. Bax BD, Chan PF, Eggleston DS, Fosberry A, Gentry DR, Gorrec $\mathrm{F}$, et al. Type IIA topoisomerase inhibition by a new class of antibacterial agents. Nature. 2010;466(7309):935-40. https:// doi.org/10.1038/natureo9197 PMID: 20686482

10. Scangarella-Oman NE, Hossain M, Dixon PB, Ingraham K, Min S, Tiffany CA, et al. Microbiological Analysis from a Phase 2 Randomized Study in Adults Evaluating Single Oral Doses of Gepotidacin in the Treatment of Uncomplicated Urogenital Gonorrhea Caused by Neisseria gonorrhoeae. Antimicrob Agents Chemother. 2018;62(12):e01221-18. https://doi. org/10.1128/AAC.01221-18 PMID: 30249694

11. Scangarella-Oman N, Hossain M, Dixon P, Ingraham K, Min S, Tiffany $C$, et al. P2.38 Microbiological analysis from a phase ii study in adults evaluating single doses of gepotidacin (GSK2140944) in the treatment of uncomplicated urogenital gonorrhoea caused by Neisseria gonorrhoeae. Sex Transm Infect. 2017;93(Suppl 2):A84.

12. Garnett GP, Mertz KJ, Finelli L, Levine WC, St Louis ME. The transmission dynamics of gonorrhoea: modelling the reported behaviour of infected patients from Newark, New Jersey. Philos Trans R Soc Lond B Biol Sci. 1999;354(1384):787-97. https:// doi.org/10.1098/rstb.1999.0431 PMID: 10365404

13. Hethcote H, Yorke J. Gonorrhea transmission dynamics and control. Berlin: Springer. 1984.

14. Whittles LK, White PJ, Didelot X. Estimating the fitness cost and benefit of cefixime resistance in Neisseria gonorrhoeae to inform prescription policy: A modelling study. PLoS Med. 2017;14(10):e1002416. https://doi.org/10.1371/journal. pmed.1002416 PMID: 29088226

15. Taylor SN, Morris DH, Avery AK, Workowski KA, Batteiger $B E$, Tiffany CA, et al. Gepotidacin for the Treatment of Uncomplicated Urogenital Gonorrhea: A Phase 2, Randomized, Dose-Ranging, Single-Oral Dose Evaluation. Clin Infect Dis. 2018;67(4):504-12. https://doi.org/10.1093/cid/ciy145 PMID: 29617982

16. Office for National Statistics (ONS). United Kingdom population mid-year estimate 2016. London: ONS. [Accessed: 25 Nov 2017]. Available from: www.ons.gov.uk/ peoplepopulationandcommunity/populationandmigration/ populationestimates/timeseries/ukpop/pop

17. Didelot X, Dordel J, Whittles LK, Collins C, Bilek N, Bishop CJ, et al. Genomic Analysis and Comparison of Two Gonorrhea Outbreaks. MBio. 2016;7(3):e00525-16. https://doi. org/10.1128/mBio.00525-16 PMID: 27353752

18. Brittain C, Childs M, Duley L, Harding J, Hepburn T, Meakin $\mathrm{G}$, et al. Gentamicin versus ceftriaxone for the treatment of gonorrhoea (G-TOG trial): study protocol for a randomised trial. Trials. 2016;17(1):558. https://doi.org/10.1186/s13063-0161683-8 PMID: 27881151

19. Bjedov I, Tenaillon O, Gérard B, Souza V, Denamur E, Radman M, et al. Stress-induced mutagenesis in bacteria. Science. 2003;300(5624):1404-9. https://doi.org/10.1126/ science.1082240 PMID: 12775833

20. Yang J, Annamalai T, Cheng B, Banda S, Tyagi R, Tse-Dinh YC. Antimicrobial Susceptibility and SOS-Dependent Increase in Mutation Frequency Are Impacted by Escherichia coli Topoisomerase I C-Terminal Point Mutation. Antimicrob Agents Chemother. 2015;59(10):6195-202. https://doi.org/10.1128/ AAC.00855-15 PMID: 26248366

21. Schook PO, Stohl EA, Criss AK, Seifert HS. The DNA-binding activity of the Neisseria gonorrhoeae LexA orthologue NG1427 is modulated by oxidation. Mol Microbiol. 2011;79(4):8466o. https://doi.org/10.1111/j.1365-2958.2010.07491.x PMID: 21299643

22. Nagel M, Reuter T, Jansen A, Szekat C, Bierbaum G. Influence of ciprofloxacin and vancomycin on mutation rate and transposition of IS256 in Staphylococcus aureus. Int J Med Microbiol. 2011;301(3):229-36. https://doi.org/10.1016/j. ijmm.2010.08.021 PMID: 21115395

23. Vestergaard M, Paulander W, Marvig RL, Clasen J, Jochumsen $\mathrm{N}$, Molin S, et al. Antibiotic combination therapy can select for broad-spectrum multidrug resistance in Pseudomonas aeruginosa. Int J Antimicrob Agents. 2016;47(1):48-55. https:// doi.org/10.1016/j.ijantimicag.2015.09.014 PMID: 26597931

24. Spratt BG, Bowler LD, Zhang QY, Zhou J, Smith JM. Role of interspecies transfer of chromosomal genes in the evolution of penicillin resistance in pathogenic and commensal Neisseria species. J Mol Evol. 1992;34(2):115-25. https://doi.org/10.1007/ BFo0182388 PMID: 1556747

25. De Silva D, Peters J, Cole K, Cole MJ, Cresswell F, Dean G, et al. Whole-genome sequencing to determine transmission of Neisseria gonorrhoeae: an observational study. Lancet Infect Dis. 2016;16(11):1295-303. https://doi.org/10.1016/S14733099(16)30157-8 PMID: 27427203

26. Grad YH, Kirkcaldy RD, Trees D, Dordel J, Harris SR, Goldstein $E$, et al. Genomic epidemiology of Neisseria gonorrhoeae with reduced susceptibility to cefixime in the USA: a retrospective observational study. Lancet Infect Dis. 2014;14(3):2206. https://doi.org/10.1016/S1473-3099(13)70693-5 PMID: 24462211

27. Ezewudo MN, Joseph SJ, Castillo-Ramirez S, Dean D, Del Rio C, Didelot X, et al. Population structure of Neisseria gonorrhoeae based on whole genome data and its relationship with antibiotic resistance. PeerJ. 2015;3:e806. https://doi. org/10.7717/peerj.806 PMID: 25780762

28. World Health Organization (WHO). Global action plan to control the spread and impact of antimicrobial resistance in Neisseria gonorrhoeae. Geneva: WHO; 2012. Available from: https:// apps.who.int/iris/handle/10665/44863

29. Babraham Institute. Babraham Bioinformatics. FastQC. Cambridge: Babraham Institute. 2012. Available from: http:// www.bioinformatics.babraham.ac.uk/projects/fastqc/

30. Li H. Aligning sequence reads, clone sequences and assembly contigs with BWA-MEM. ArXiv13033997 Q-Bio. 2013. Available from: https://arxiv.org/abs/1303.3997

31. Walker BJ, Abeel T, Shea T, Priest M, Abouelliel A, Sakthikumar $S$, et al. Pilon: an integrated tool for comprehensive microbial variant detection and genome assembly improvement. PLoS One. 2014;9(11):e112963. https://doi.org/10.1371/journal. pone.0112963 PMID: 25409509

32. Harris SR, Cole MJ, Spiteri G, Sánchez-Busó L, Golparian D, Jacobsson $\mathrm{S}$, et al. Public health surveillance of multidrugresistant clones of Neisseria gonorrhoeae in Europe: a genomic survey. Lancet Infect Dis. 2018;18(7):758-68. https://doi. org/10.1016/S1473-3099(18)30225-1 PMID: 29776807

33. Grad YH, Harris SR, Kirkcaldy RD, Green AG, Marks DS, Bentley $\mathrm{SD}$, et al. Genomic Epidemiology of Gonococcal Resistance to Extended-Spectrum Cephalosporins, Macrolides, and Fluoroquinolones in the United States, 2000-2013. J Infect Dis. 2016;214(10):1579-87. https://doi.org/10.1093/infdis/jiw420 PMID: 27638945

34. Levin BR, Bonten MJ. Cycling antibiotics may not be good fo your health. Proc Natl Acad Sci USA. 2004;101(36):13101-2. https://doi.org/10.1073/pnas.0404970101 PMID: 15340145

35. Tuite AR, Gift TL, Chesson HW, Hsu K, Salomon JA, Grad YH. Impact of Rapid Susceptibility Testing and Antibiotic Selection Strategy on the Emergence and Spread of Antibiotic Resistance in Gonorrhea. J Infect Dis. 2017;216(9):1141-9. https://doi. org/10.1093/infdis/jix450 PMID: 28968710

36. Fingerhuth SM, Low N, Bonhoeffer S, Althaus CL. Detection of antibiotic resistance is essential for gonorrhoea pointof-care testing: a mathematical modelling study. BMC Med. 2017;15(1):142. https://doi.org/10.1186/s12916-017-0881-x PMID: 28747205

37. Frénoy A, Bonhoeffer S. Death and population dynamics affect mutation rate estimates and evolvability under stress in bacteria. bioRxiv. 2017. https://doi.org/10.1101/224675

38. Obolski U, Hadany L. Implications of stress-induced genetic variation for minimizing multidrug resistance in bacteria. BMC Med. 2012;10(1):89. https://doi.org/10.1186/1741-7015-10-89 PMID: 22889082

License, supplementary material and copyright 
This is an open-access article distributed under the terms of the Creative Commons Attribution (CC BY 4.0) Licence. You may share and adapt the material, but must give appropriate credit to the source, provide a link to the licence and indicate if changes were made.

Any supplementary material referenced in the article can be found in the online version.

This article is copyright of the authors or their affiliated institutions, 2020. 